\section{Esophageal tuberculosis}

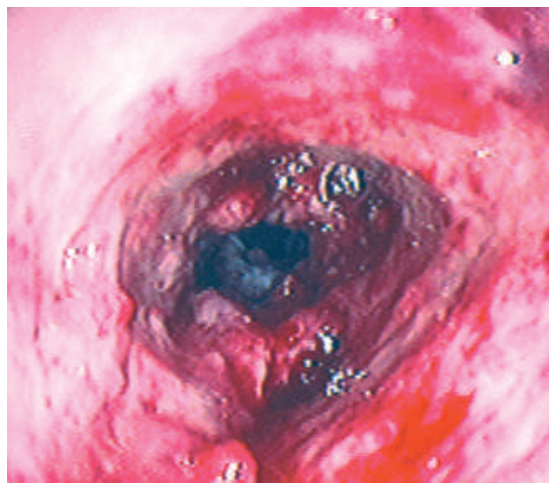

Figure 1 Endoscopic image showing a hypertrophic growth in the lumen of the esophagus.

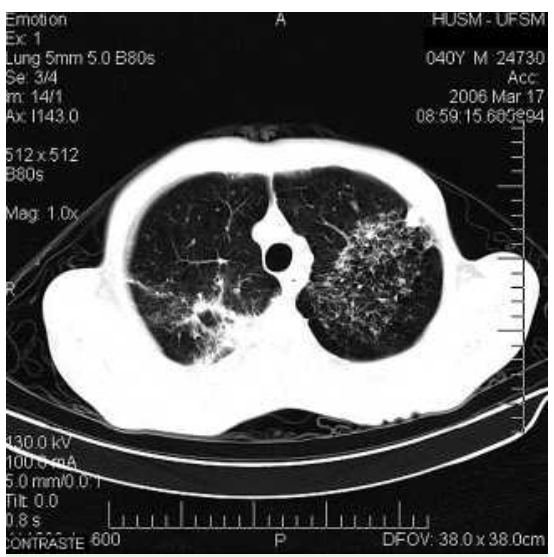

Figure 2 Computed tomographic image showing pulmonary tuberculosis.



Figure 3 Photomicrograph of the esophageal biopsy tissue stained by the Ziehl-Neelsen method (original magnification $\times 200$ ).

Esophageal tuberculosis is very rare. Most of the reported cases have been secondary to pulmonary tuberculosis. With the increased incidence of tuberculous infection linked to AIDS, it is important to be aware of this condition [1]. Esophageal tuberculosis may present in three forms at upper gastrointestinal endoscopy: ul-

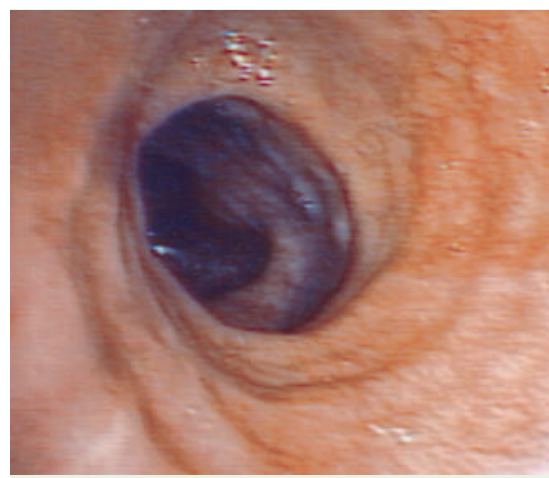

Figure 4 Endoscopic view 45 days after the patient started antituberculosis therapy.

cerative, hyperplastic, or granular. The ulcerative form presents as ulcers with irregular margins and membranous necrotic bases. The hypertrophic form occurs as a consequence of fibrosis of the esophageal wall with a pseudotumoral presentation, which can be difficult to distinguish from a malignancy. The granular form presents as small and verrucous grayish nodules with some ulceration $[1,2]$.

We report here the case of a 40-year-old black man whose chief complaint was of progressive dysphagia and a 7-kg weight loss over a 3-month period. He had a 40 pack-years smoking history and a chronic cough. On physical examination, he appeared chronically ill and showed evidence of weight loss. No abnormality was found in the chest and abdominal examinations. Upper gastrointestinal endoscopy revealed an infiltrative growth with stricture formation and ulceration, extending from $18 \mathrm{~cm}$ to $23 \mathrm{~cm}$ from the incisors ( $\bullet$ Figure 1 ), an appearance that was suggestive of esophageal cancer. However, histological examination of the biopsied tissue showed inflammation and necrosis with no sign of malignancy. Because the chest computed tomographic scan was suggestive of pulmonary tuberculosis ( $\bullet$ Figure 2 ), the esophageal biopsies were stained with a Ziehl-Neelsen stain, and found to be positive for acidfast bacilli ( $\bullet$ Figure 3). Bronchial washings obtained by bronchoscopy demonstrated Mycobacterium tuberculosis organisms. The purified protein derivative skin test (PPD-S) and HIV test were negative. The patient was treated with a three- drug regimen of rifampicin, isoniazid, and pyrazinamide, and his good response to the antituberculosis therapy after 45 days of treatment confirmed the diagnosis and the decision to continue maintenance treatment with the standard therapy (๑ Figure 4).

Endoscopy_UCTN_Code_CCL_1AB_2AC_3AZ

\section{R. B. Fagundes ${ }^{1,2}$, R. P. Dalcin ${ }^{1}$, M. P. Rocha ${ }^{3}$, C. C. Moraes ${ }^{1}$, V. S. Carlotto ${ }^{1}$, M. O. Wink ${ }^{1}$}

1 Serviço de Gastroenterologia do Hospital Universitario, Universidade Federal de Santa Maria, Santa Maria, RS, Brazil

2 Programa de Pos-Graduacao: Gastroenterologia, Faculdade de Medicina, Universidade Federal do Rio Grande do Sul, Porto Alegre, RS, Brazil

Serviço de Patologia da Universidade Federal de Santa Maria, Santa Maria, RS, Brazil

\section{References}

1 Jain SK, Jain S, Jain M, Yaduvanshi A. Esophageal tuberculosis: is it so rare? Report of 12 cases and review of the literature. Am J Gastroenterol 2002; 97: 287-291

2 Burch WC. Esophageal tuberculosis. Am J Gastroenterol 1992; 87: 929

\section{Bibliography}

DOI $10.1055 / s-2007-966152$

Endoscopy 2007; 39: E149

(c) Georg Thieme Verlag KG Stuttgart · New York . ISSN 0013-726X

\section{Corresponding author \\ R. B. Fagundes, MD}

Avenida Nilo Pecanha 2863

ap $804-91330-001$

Porto Alegre

RS

Brazil

Fax: 5133085616

rfagundes@pro.viars.com.br 